\title{
REFERENCES
}

1. L. Carlitz, Some cyclotomic determinants, Bull. Calcutta Math. Soc. vol. 49 (1957) pp. 49-51.

2. Emma Lehmer, On a resultant connected with Fermat's last theorem, Bull. Amer. Math. Soc. vol. 41 (1935) pp. 864-867.

DUKE UNIVERSITY

\section{ON THE MEASURE OF HILBERT NEIGHBORHOODS FOR PROCESSES WITH STATIONARY, INDEPENDENT INCREMENTS}

GLEN BAXTER ${ }^{1}$

1. Introduction. Let $\{x(t), 0 \leqq t<\infty\}$ denote a stochastic process with stationary, independent increments for which $x(0)=0$. According to the Lévy-Khitchine representation, the characteristic function of $x(t)$ has the form

$$
E\left\{e^{i \xi x(t)}\right\}=e^{-t \psi(\xi)}
$$

Moreover,

$$
\psi(\xi)=-i \gamma \xi-\int_{-\infty}^{\infty}\left(e^{i \xi u}-1-\frac{i \xi u}{1+u^{2}}\right) \frac{1+u^{2}}{u^{2}} d G(u),
$$

where $G(u)$ is a bounded, nondecreasing function with $G(-\infty)=0$ and where $\gamma$ is a real-valued constant. Below it is shown that for certain processes of this type the measure of the Hilbert neighborhood of the origin is related to the solution of a certain differential system. In fact, (A) if $\{x(t), 0 \leqq t<\infty\}$ is a separable stochastic process with symmetric, stationary, and independent increments for which $x(0)=0$, and if

Received by the editors February 24, 1958 and, in revised form, December 8, 1958.

1 This research was supported by the United States Air Force, through the office of Scientific Research of the Air Research and Development Command, under contract No. AF 18 (603)-30. Reproduction in whole or in part is permitted for any purpose of the United States Government. 


$$
\phi(\xi)=\int_{0}^{\infty} e^{-s t} E\left\{\exp \left[-u \int_{0}^{t} x^{2}(\tau) d \tau+i \xi x(t)\right]\right\} d t
$$

then

$$
\phi(\xi)=\int_{-\infty}^{\infty} \tilde{\phi}(x, \xi) d x,
$$

where $\phi(x) \equiv \tilde{\phi}(x, \xi)$ is the unique solution of the system

$$
\begin{aligned}
& u \tilde{\phi}^{\prime \prime}-[s+\psi(x)] \tilde{\phi}=0, \\
& \tilde{\phi} \rightarrow 0 \text { as } x \rightarrow \pm \infty, \\
& \tilde{\phi} \text { is continuous for all } x, \\
& \tilde{\phi}^{\prime} \text { is continuous except at } x=\xi, \\
& \lim _{x \rightarrow \xi} \tilde{\phi}^{\prime}-\lim _{x \rightarrow \xi^{+}} \tilde{\phi}^{\prime}=1 / u .
\end{aligned}
$$

In other words, $\tilde{\phi}(x, \xi)$ is the Green's function of the differential system formed from the first two lines in (5).

One might ask what connection the $x$ variable in (5) has with the process $\{x(t), 0 \leqq t<\infty\}$ since this variable is integrated out in (4). No "simple" interpretation of $\tilde{\phi}(x, \xi)$ in terms of the process seems to exist. In some cases it can be shown that $\phi(\xi)$ itself satisfies a differential equation. Specifically, (B) ${ }^{2}$ if $\{x(t), 0 \leqq t<\infty\}$ is a separable stochastic process with stationary, independent increments for which $x(0)=0$, and if $E\left\{x^{2}(t)\right\}$ exists for all $t$ and has a finite Laplace transform, then the $\phi(\xi)$ in (3) satisfies

$$
u \phi^{\prime \prime}(\xi)-[s+\psi(\xi)] \phi(\xi)=-1 .
$$

The boundary conditions for $\phi(\xi)$ may vary from process to process. For processes with density functions it is clear from (3) that $\phi(\xi) \rightarrow 0$ as $\xi \rightarrow \pm \infty$. Later, we give an example where $\phi(\xi)$ is periodic of period $2 \pi$ in $\xi$ (see $\S 4$ ).

2. Proof of (A). Simply described, the proof is based on a change of order of integration, both at the real line and the function space level. However, since the integrals over the real line do not exist in the ordinary sense, they are replaced by Riemann sums in the proof. We denote by $\{x(t), 0 \leqq t<\infty\}$ the original process, by $\{w(t), 0 \leqq t<\infty\}$ the Wiener process, and by $E_{I}\{\ldots\}$ and $E_{W}\{\ldots\}$, respectively, the expectations on these processes. For any $t$, let $x_{n k}=x(k t / n)$ and $w_{n k}=w(k t / n)$. Then,

${ }^{2}$ Darling has mentioned to the author that result (B) follows from a previous paper by Siegert and Darling [2]. 


$$
\begin{aligned}
& E_{I}\left\{\exp \left[-u \frac{t}{n} \sum_{k=0}^{n-1} x_{n k}^{2}+i \xi x_{n n}\right]\right\} \\
& (7)=E_{I}\left\{E_{W}\left\{\exp \left[i(2 u)^{1 / 2} \sum_{k=0}^{n-1} x_{n k}\left(w_{n, n-k}-w_{n, n-k-1}\right)+i \xi x_{n n}\right]\right\}\right\} \\
& =E_{I}\left\{E_{W}\left\{\exp \left[i \sum_{k=1}^{n}\left((2 u)^{1 / 2}\left(w_{n, n \cdot k}+\xi\right)\left(x_{n k}-x_{n, k-1}\right)\right]\right\}\right\}\right. \\
& =E_{W}\left\{\exp \left[-\frac{t}{n} \sum_{k=1}^{n} \psi\left((2 u)^{1 / 2} w_{n, n-k}+\xi\right)\right]\right\} .
\end{aligned}
$$

Passing to the limit on $n$ in (7) and using separability, we have

$$
\begin{aligned}
E_{I}\left\{\operatorname { e x p } \left[-u \int_{0}^{t} x^{2}(\tau) \mathrm{d} \tau+\right.\right. & i \xi x(t)]\} \\
& =E_{W}\left\{\exp \left[-\int_{0}^{t} \psi\left((2 u)^{1 / 2} w(\tau)+\xi\right) d \tau\right]\right\} .
\end{aligned}
$$

We deduce that

$$
\phi(\xi)=\int_{0}^{\infty} e^{-s t} E_{W}\left\{\exp \left[-\int_{0}^{t} \psi\left((2 u)^{1 / 2} w(\tau)+\xi\right) d \tau\right]\right\} d t .
$$

The result of $\mathrm{Kac}[1, \S 3]$ can be used to evaluate the right-hand side of (8) since $\psi$ is a non-negative function. Thus,

$$
\phi(\xi)=\int_{-\infty}^{\infty} \tilde{\phi}(y, 0) d y,
$$

where $\tilde{\phi}(y, \eta)$ is the unique Green's function of the system

$$
\begin{aligned}
\frac{1}{2} \tilde{\phi}^{\prime \prime}-\left[s+\psi\left((2 u)^{1 / 2} y+\xi\right)\right] \tilde{\phi} & =0, \\
\tilde{\phi} & \rightarrow 0 \text { as } y \rightarrow \pm \infty .
\end{aligned}
$$

A change of variable to $x=(2 u)^{1 / 2} y+\xi$ now gives the desired result.

3. Proof of (B). Let $F_{t}(x)$ be the distribution function of $x(t)$ and let

$$
V_{a}(x)= \begin{cases}x^{2} & |x| \leqq a, \\ a^{2} & |x| \geqq a .\end{cases}
$$

Starting with 


$$
\begin{aligned}
\tilde{Q}_{0}(x, t) & =F_{t}(x), \\
\widetilde{Q}_{n+1}(x, t) & =\int_{0}^{t} \int_{-\infty}^{\infty} F_{t-\tau}(x-\xi) V_{a}(\xi) d_{\xi} Q_{n}(\xi, \tau) d \tau,
\end{aligned}
$$

and following exactly the method of $\mathrm{Kac}[1, \S 3]$, it is possible to show that the function

(9) $\quad q_{a}(x)=\int_{0}^{\infty} e^{-s t} E_{I}\left\{\exp \left[-u \int_{0}^{t} V_{a}(x(\tau)) d \tau\right], x(t)<x\right\} d t$

satisfies the equation

$$
q_{a}(x)+u \int_{-\infty}^{\infty} F(x-\xi) V_{a}(\xi) d_{\xi} q_{a}(\xi)=F(x),
$$

where

$$
F(x)=\int_{0}^{\infty} e^{-s t} F_{t}(x) d t
$$

From (9) we see that $q_{a}(x)$ is a decreasing function of $a$. Let $q(x)$ be the limit of $q_{a}(x)$ as $a$ becomes infinite. From (9) and (10) it follows that for any $x>y$

$$
q(x)-q(y) \leqq q_{a}(x)-q_{a}(y) \leqq F(x)-F(y),
$$

so that for any $A>0$

$$
\int_{|\xi|>A} \xi^{2} d q(\xi) \leqq \int_{|\xi|>A} \xi^{2} d q_{a}(\xi) \leqq \int_{|\xi|>A} \xi^{2} d F(\xi) .
$$

The assumption that $E_{I}\left\{x^{2}(t)\right\}$ exists and has a finite Laplace transform implies that the right-hand estimate in (12) goes to zero as $A$ becomes infinite. Thus, we can show

$$
\lim _{a \rightarrow \infty} \int_{-a}^{a} F(x-\xi) \xi^{2} d q_{a}(\xi)=\int_{-\infty}^{\infty} F(x-\xi) \xi^{2} d q(\xi) .
$$

Taking limits on $a$ in (10) gives finally

$$
q(x)+u \int_{-\infty}^{\infty} F(x-\xi) \xi^{2} d q(\xi)=F(x) .
$$

Now, we observe from (3) and (9) that

$$
\phi(\xi)=\int_{-\infty}^{\infty} e^{i \xi x} d q(x),
$$


and from (12) it follows that $\phi^{\prime \prime}(\xi)$ exists. Furthermore, from (11)

$$
\int_{-\infty}^{\infty} e^{i \xi x} d F(x)=\int_{0}^{\infty} e^{-s t-t \psi(\xi)} d t=1 /(s+\psi(\xi)) .
$$

Taking Fourier transforms throughout (13), we find after a simple algebraic manipulation

$$
u \phi^{\prime \prime}(\xi)-[s+\psi(\xi)] \phi(\xi)=-1 .
$$

4. Examples. We illustrate the results with two examples.

Cauchy process. In this case $\psi(x)=|x|$ and (A) applies. We want the Green's function of the system.

$$
\left\{\begin{array}{l}
u \phi^{\prime \prime}-(s+|x|) \phi=0 \\
\phi \rightarrow 0 \text { as } x \rightarrow \pm \infty
\end{array}\right.
$$

It is not difficult to show that for the $\phi(\xi)$ in (3) in this case

$$
\begin{aligned}
\phi(0) & =\int_{0}^{\infty} e^{-s t} E_{I}\left\{\exp \left[-u \int_{0}^{t} x^{2}(\tau) d \tau\right]\right\} d t \\
& =\frac{1}{s K_{2 / 3}\left(\frac{2}{3}\left(\frac{s^{3}}{u}\right)^{1 / 2}\right)} \int_{(2 / 3)\left(s^{3} / u\right)^{1 / 2}}^{\infty} K_{1 / 3}(x) d x
\end{aligned}
$$

where $K_{p}(x)$ is the modified Bessel function of the second kind. We are not able to invert this transform in closed form. Another form for $\phi(0)$ and an inversion with respect to $s$ appear in [2].

Poisson process. ${ }^{3}$ For this case $\psi(x)=a(1-\exp (i x))$ and (B) applies. Now set

$$
A_{k}=\int_{0}^{\infty} e^{-s t} E_{I}\left\{\exp \left[-u \int_{0}^{t} x^{2}(\tau) d \tau\right], x(t)=k\right\} d t
$$

so that

$$
\phi(\xi)=\sum_{k=0}^{\infty} A_{k} e^{i k \xi}
$$

From (6)

$$
\sum_{k=0}^{\infty}\left(u k^{2}+s+a\right) A_{k} e^{i k \xi}=1+a \sum_{k=0}^{\infty} A_{k} e^{i(k+1) \xi}
$$

Thus,

${ }^{3}$ Darling has mentioned to the author that this calculation can also be made using results by Darling [3]. 


$$
\begin{aligned}
A_{n} & =a^{n} /\left(\prod_{k=0}^{n}\left(s+a+u k^{2}\right)\right) \\
& =\sum_{k=0}^{n} \frac{a^{n}}{u^{n}\left(s+a+u k^{2}\right)}\left(\prod_{m=0 ; m \neq k}^{n} \frac{1}{m^{2}-k^{2}}\right) .
\end{aligned}
$$

Inverting (14) with respect to $s$ we find

$$
E_{I}\left\{\exp \left[-u \int_{0}^{t} x^{2}(\tau) d \tau\right], x(t)=n\right\}
$$

$$
=\sum_{k=0}^{n} \frac{e^{-a t-u t k^{2}}}{u^{n}} a^{n}\left(\prod_{m=0 ; m \neq k}^{n} \frac{1}{m^{2}-k^{2}}\right),
$$

and inverting (15) with respect to $u$ we have

$$
\begin{aligned}
& P\left\{\int_{0}^{t} x^{2}(\tau) d \tau\right.<\alpha, x(t)=n\} \\
&=e^{-a t} \frac{a^{n}}{n !} \sum_{k=0}^{\min \left\{n,(\alpha / t)^{1 / 2}\right\}}\left(\alpha-t k^{2}\right)^{n}\left(\prod_{m=0 ; m \neq k}^{n} \frac{1}{m^{2}-k^{2}}\right) .
\end{aligned}
$$

It is interesting to note that if $n<(\alpha / t)^{1 / 2}$ and if $x(t)$ is a sample function for which $x(t)=n$, then necessarily

$$
\int_{0}^{t} x^{2}(\tau) d \tau<\alpha
$$

and the left-hand side of (16) becomes merely $P\{x(t)=n\}$ $=e^{-a t}(a t)^{n} / n$ !. Thus, we have the following rather unusual identity,

$$
\sum_{k=0}^{n}\left(\alpha-t k^{2}\right)^{n}\left(\prod_{m=0 ; m \neq k}^{n} \frac{1}{m^{2}-k^{2}}\right) \equiv t^{n}
$$

\section{REFERENCES}

1. M. Kac, On some connections between probability theory and differential and integral equations, Proceedings of the Second Berkeley Symposium, 1951, pp. 189-215.

2. - On the average of a certain Wiener functional and a related limit theorem in calculus of probability, Trans. Amer. Math. Soc. vol. 59 (1946) pp. 401-414.

3. D. A. Darling and A. J. F. Siegert, On the distribution of certain functionals of Markov chains and processes, Proc. Nat. Acad. Sci. U.S.A. vol. 42 (1956) pp. 525529.

4. D. A. Darling, On a class of problems related to the random division of an interval, Ann. Math. Statist. vol. 24 (1953) pp. 239-253. 